\title{
VISITA PRÉ-OPERATÓRIA DE ENFERMAGEM: PROPOSTA METODOLÓGICA FUNDAMENTADA NO MODELO CONCEITUAL DE LEVINE
}

\author{
PRE-OPERATIVE NURSING VISIT: METHODOLOGIC PROPOSAL BASED ON LEVINE'S CONCEPTUAL \\ MODEL
}
VISITA PREOPERATÓRIA DE ENFERMERÍA: PROPOSTA METODOLÓGICA FUNDAMENTADA EN EL MODELO CONCEPTUAL DE LEVINE

\section{Marister Piccoli ${ }^{1}$, Cristina Maria Galvão ${ }^{2}$}

RESUMO: O presente estudo teve como objetivo elaborar um roteiro de coleta de dados na forma de visita pré-operatória voltado para a identificação dos diagnósticos de enfermagem no período transoperatório de pacientes submetidos à cirurgia geral. $\mathrm{O}$ referencial teórico selecionado foi o modelo conceitual de Levine, tendo como eixo norteador os quatros princípios de conservação, a saber: conservação de energia, conservação da integridade estrutural, conservação da integridade pessoal e conservação da integridade social.

PALAVRAS CHAVES: Enfermagem Perioperatória; Cuidados Pré-Operatórios; Filosofia em Enfermagem.

ABSTRACT: This study aimed to elaborate a script for data colleting as a form to preoperative care visit towards the nursing diagnosis identification in the trans-operative of clients submitted to general surgery. The selected theoretical framework was Levine's conceptual model based on the four conservation principles, as energy conservation, structural integrity conservation, personal integrity conservation and social integrity conservation.

KEYWORDS: Perioperative Nursing; Preoperative Care; Nursing Philosophy.

RESUMEN: El presente estudio tuvo como objetivo elaborar una escritura para la recolección de datos en la forma de la visita diaria preoperatorio dirigido hacia la identificación del diagnostico de enfermería en el período transoperatorio de pacientes sometidos a cirugía general. El referencial teórico seleccionado fue el modelo conceptual de Levine, teniendo como eje orientador los cuatros principios de conservación: conservación de energía, conservación de integridad estructural, conservación de integridad personal y conservación de integridad social.

TERMINOS CLAVES: Enfermería Perioperatoria; Cuidados Preoperatorios; Filosofia en Enfermería.
${ }^{1}$ Enfermeira, Doutoranda na EERP/USP/Enfermagem Fundamental, Docente da Universidade Estadual do Oeste do Paraná. Cascavel, Paraná.

${ }^{2}$ Enfermeira, Professor Associado da Escola de Enfermagem de Ribeirão Preto, da Universidade de São Paulo, Centro Colaborador da OMS para o desenvolvimento da pesquisa em enfermagem. Ribeirão Preto, São Paulo. E-mail: crisgalv@eerp.usp.br 
PICCOLI, Marister; GALVÃO, Cristina Maria. BIOÉTICA - UM ENSAIO SOBRE SUA INSERÇÃO NOS CURSOS DE GRADUAÇÃO EM ENFERMAGEM. Revista Eletrônica de Enfermagem, v. 07, n. 03, p. 366 - 372, 2005. Disponível em http://www.fen.ufg.br/Revista/revista7 3/atualizacao.htm

De acordo com CASTELLANOS \& MANDELBAUM (1985), para a utilização da sistematização da assistência o enfermeiro deve levar em consideração dois componentes básicos: um modelo conceitual ou um guia que levará o enfermeiro à que dados coletar para identificar os diagnósticos de enfermagem e a conseqüente implementação do plano de cuidados; outro componente refere-se ao conhecimento cognitivo e afetivo necessários ao atendimento do paciente com necessidades afetadas devido ao procedimento anestésico-cirúrgico.

CASTELLANOS (1978) em um estudo sobre a aplicação do processo de enfermagem na unidade de centro cirúrgico, refere à visita pré-operatória de enfermagem como o primeiro item da avaliação do paciente cirúrgico, procedimento este indispensável tanto no preparo físico quanto no emocional. A visita pré-operatória de enfermagem é o início da sistematização da assistência de enfermagem perioperatória.

A assistência de enfermagem ao paciente no período trans-operatório é relativa ao desenvolvimento das ações de enfermagem já planejadas e implementadas desde a recepção do paciente na unidade de centro cirúrgico até a saída deste para a sala de recuperação pós anestésica, esta fase compreende uma das etapas da sistematização da assistência de enfermagem perioperatória e só pode ser realizada com a devida efetivação da primeira fase, o que consiste na visita pré-operatória, onde o enfermeiro coleta dados por meio da visita ao paciente, consulta no prontuário e também busca informações com outros profissionais da equipe de saúde, ou seja, realiza a avaliação pré-operatória, identificação dos problemas ou diagnósticos de enfermagem e elaboração do plano de cuidados.

A nosso ver, a identificação dos diagnósticos de enfermagem na visita pré-operatória é fundamental para o enfermeiro elaborar e implementar o plano de cuidados de enfermagem para o período transoperatório pois, sabemos que vários são os aspectos que irão influenciar a resposta do paciente ao procedimento anestésico cirúrgico. Assim, o presente estudo teve como objetivo elaborar um instrumento de visita pré-operatória de enfermagem, fundamentado no modelo conceitual de Levine voltado à identificação dos diagnósticos de enfermagem no período transoperatório.

\section{REFERENCIAL TEÓRICO}

\section{Modelo Conceitual de Myra Strin Levine}

Muitas teorias foram propostas na enfermagem, mas, considerando o paciente cirúrgico, primamos por um referencial teórico que nos oferecesse uma visão total do ser humano.

Optamos então pelo modelo conceitual de Myra Strin Levine, pois esta autora caracteriza o homem como um todo dinâmico, em constante interação com o ambiente; além deste ponto, este modelo preocupa- se com o paciente que adentra em um estabelecimento de saúde necessitando de assistência, ou seja, com estado de saúde alterado. Essas mesmas características são encontradas no paciente cirúrgico.

Em seu modelo conceitual, Levine desenvolveu quatro princípios de conservação: de energia, da integridade estrutural, da integridade pessoal e da integridade social do paciente. Para LEONARD (1993) o modelo conceitual de Levine centraliza-se na intervenção da enfermagem, na adaptação e na reação dos pacientes à doença.

De acordo com LEONARD (1993), Levine entende que o ser humano deve ser visto no todo, o que leva o indivíduo a um ser complexo que é dependente de sua relação com os outros, as dimensões dessa dependência está ligada com os quatro princípios de conservação, e que esta dependência existe em todas as passagens de sua existência, na sobrevivência; afirma também que o enfermeiro deve estar consciente dessa dependência e estar preparado para atuar na transformação que o estresse causado por algum desequilíbrio possa alterar o funcionamento do organismo humano. Levine acredita que o enfermeiro deve assumir a ajuda ao paciente para transformá-lo e auxiliá-lo na adaptação às mudanças oriundas da doença.

Levine entende enfermagem como uma disciplina, o atendimento de enfermagem é um processo no qual as intervenções estão baseadas na avaliação, utilização dos princípios de conservação, reconhecimento de mudanças comportamentais; está centrado em um método científico e promove cuidado integral (LEONARD, 1993).

A meta da enfermagem, segundo LEVINE (1973) é manter ou recuperar uma pessoa para um estado de saúde, através dos princípios de conservação. A conservação da energia que consiste no primeiro princípio, refere-se ao equilíbrio entre a energia de saída e a energia de entrada, com o propósito de evitar cansaço excessivo utilizando repouso, nutrição e exercícios adequados. A autora acrescenta que a habilidade do corpo humano, é dependente do seu balanço de energia, e a energia exigida pelas alterações fisiológicas durante a doença, representa uma exigência adicional nos sistemas de produção para a cura.

O segundo princípio, conservação da integridade estrutural, refere-se à manutenção ou recuperação da estrutura do corpo, ou seja, a prevenção do colapso físico e a promoção da cura. Em muitos casos conforme relata Levine, as pessoas estão expostas a fatores ameaçadores no meio ambiente para infligir ferimentos, o corpo processa um número de sistemas de defesa eficientes que o protegem da perda de fluídos, introdução de microorganismos, habilidade para locomoção, adaptações rápidas nas mudanças de temperaturas externas, umidade e outras. Somente o organismo intacto pode mover-se com liberdade e sem restrições no meio. 
PICCOLI, Marister; GALVÃO, Cristina Maria. BIOÉTICA - UM ENSAIO SOBRE SUA INSERÇÃO NOS CURSOS DE GRADUAÇÃO EM ENFERMAGEM. Revista Eletrônica de Enfermagem, v. $07, \quad$. $03, \quad$ p. 366 - $372, \quad 2005$. Disponível em http://www.fen.ufg.br/Revista/revista7 3/atualizacao.htm

O terceiro princípio, conservação da integridade pessoal, refere-se à manutenção ou recuperação da identidade e auto-estima do paciente, sendo que para Levine o senso de identidade é a mais completa evidência de totalidade. Para a autora, existe sempre uma parcela da vida das pessoas que é dividida através de experiência comum, mas a decisão de dividir ou não é sempre uma expressão da privacidade de alguém, relata também que a entrada de uma pessoa no hospital tem sempre significado um sacrifício da integridade pessoal, outros tomarão decisões, que no passado o paciente fazia sozinho.

O último princípio, conservação da integridade social, consiste no reconhecimento do paciente como um ser social, envolve a interação humana, particularmente aquelas que são importantes ao paciente. Levine menciona que a doença é freqüentemente solitária, e nas horas estressantes, as interações com outras pessoas tornam-se mais importantes, não somente o paciente continua envolvido nas preocupações das outras pessoas, como também novos problemas com a hospitalização podem ser resolvidos pela participação de todos que são incluídos em sua vida social. Assim, as forças das relações humanas são necessárias e para Levine consiste falha não considerar a família e os amigos do paciente.

Para Levine, conservação significa manter a unicidade ou equilíbrio adequado e o propósito da conservação é a manutenção da integridade da pessoa. Assim, considerando que o paciente submetido ao procedimento anestésico cirúrgico sofre mudanças bruscas em sua totalidade e requer uma adaptação favorável para o restabelecimento de seu estado de saúde, selecionamos este modelo para fundamentarmos nosso estudo.

\section{PROCEDIMENTO METODOLÓGICO}

\section{Construção e validação do instrumento:}

A elaboração do instrumento de coleta de dados que teve como eixo norteador os quatro princípios de conservação do modelo conceitual de Levine. A literatura pesquisada e a experiência profissional das pesquisadoras também nortearam a construção do instrumento.

No referido instrumento foi realizado a validação aparente e de conteúdo onde solicitamos a quatro especialistas (docentes da Escola de Enfermagem de Ribeirão Preto, Universidade de São Paulo, com experiência na temática em questão ou validação de instrumento) a análise do instrumento quanto aos itens abordados, forma de apresentação e verificação sobre o alcance do objetivo traçado.

Após esta fase, com a finalidade de obtermos subsídios para verificarmos a adequação do conteúdo e melhor forma de aplicação do instrumento, realizamos um teste com o objetivo de aproximação com a unidade hospitalar e sua equipe, bem como, avaliar o tempo gasto nas entrevistas e promover os ajustes necessários no instrumento. Foram entrevistados cinco pacientes, e após, fizemos as adequações necessárias, sendo que foram apenas na forma de abordagem ao paciente e sua família e procedência dos pacientes.

\section{Aplicação do Instrumento:}

O Instrumento foi utilizado na visita préoperatória ao paciente cirúrgico adulto que foi submetido a procedimento de cirurgia geral de pequeno e médio porte, no momento da coleta de dados para o estudo do qual este instrumento fez parte (PICOLI, 2000) e está sendo utilizado como a primeira etapa da sistematização da assistência de enfermagem da disciplina Enfermagem Perioperatória na Universidade Estadual do Oeste do Paraná.

\section{VISITA PRÉ-OPERATÓRIA DE ENFERMAGEM FUNDAMENTADA NO MODELO CONCEITUAL DE LEVINE (PICCOLI;GALVÃO, 2000)}

\section{Dados de identificação:}

1.1 Nome:. Idade:.........Sexo:.........Estado civil Grau Profissão:

Enfermaria: Leito:

Clínica:

Endereço

residencial:

Cidade de origem:

..Telefone 
PICCOLI, Marister; GALVÃO, Cristina Maria. BIOÉTICA - UM ENSAIO SOBRE SUA INSERÇÃO NOS CURSOS DE GRADUAÇÃO EM ENFERMAGEM. Revista Eletrônica de Enfermagem, v. 07, $n$. $03, \quad$ p. 366 - $372, \quad 2005 . \quad$ Disponível em http://www.fen.ufg.br/Revista/revista7 3/atualizacao.htm

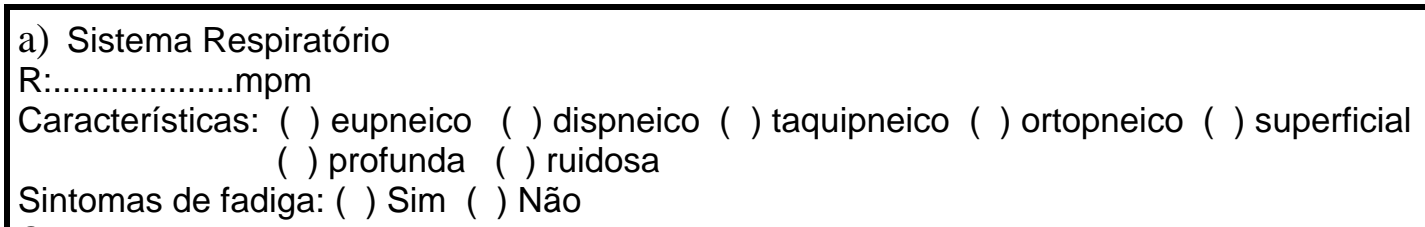

Quais:

b) Sistema Gastrintestinal

Somatometria: Peso:......... Kg Altura:............m

IMC: $\frac{P}{A 2} \quad$ ( ) Normal ( ) Sobrepeso ( ) Obeso

Características abdominais: ( ) plano ( ) distendido ( ) globoso ( ) escavado

Presença de prótese dentária: ( ) sim ( ) não

Jejum: ( ) Sim ( ) Não

Defecação: Tipo:.

Freqüência:

Apetite: ( ) Diminuído ( ) Aumentado

Observações:

C) Sistema Cardiovascular:

F.C.:...........bpm P.A :....................mmHg

Pulso : ( ) rítmico ( ) arritmico ( ) taquicardico ( ) bradicardico ( ) cheio ( ) fino

d) Sistema Músculo Esquelético

Deambulação: ( ) preservada ( ) prejudicada

Especificar:.

e) Sistema Genito-urinário e Reprodutor:

Características da urina:

Coloração:....................Freqüência:

Características do ciclo menstrual:

f) Sistema Tegumentar

T................... $\mathrm{C} \quad$ ( ) axilar ( ) oral ( ) retal

Integridade cutânea: ( ) sim ( ) não

Observações.

g) Sistema Imunológico:

Alergias: ( ) sim ( ) não ( ) não sabe

Quais:.

Vacinações: ( ) anti-hepatite B ( ) anti-tetânica

h) Sistema Neurológico

Sono: ( ) 8 horas ( )<8 horas ( ) outro padrão Especificar

alteração:

Orientação tempo/espaço : ( ) sim ( ) não

Acuidade auditiva e

visual:.

Dor: ( ) presente ( ) ausente

Local:. ..Tipo:.......

\subsection{Dados relacionados ao princípio de conservação da integridade estrutural}

1. Fatores de risco relacionados ao paciente cirúrgico

Cirurgia

proposta:

Data:

Hora: 
PICCOLI, Marister; GALVÃO, Cristina Maria. BIOÉTICA - UM ENSAIO SOBRE SUA INSERÇÃO NOS CURSOS DE GRADUAÇÃO EM ENFERMAGEM. Revista Eletrônica de Enfermagem, v. 07, $n$. $03, \quad$ p. 366 - $372, \quad 2005 . \quad$ Disponível em http://www.fen.ufg.br/Revista/revista7 3/atualizacao.htm

Anestesia

proposta:

Problemas apresentados em procedimentos anestésico cirúrgicos

anteriores:

Procedimentos invasivos:

Sondas: ( ) Sim ( ) Não

Especificar:.

Soroterapia: ( ) Sim ( ) Não

Especificar:

Tricotomia: ( ) Sim ( ) Não

Especificar:

Lavagem Intestinal: ( ) Sim ( ) Não

2. Fatores de risco individuais:

Diagnóstico médico:

Utilização de medicamentos: ( ) Sim ( ) Não

Especificar:.

Utilização de antibióticos: ( ) Sim ( ) Não

Especificar:.

Potencial de contaminação da cirurgia atual: ( ) limpa ( ) potencialmente contaminada

Tabagismo: ( ) Sim ( ) Não

( ) contaminada ( ) infectada

Quantidade:............................

\subsection{Dados relacionados ao princípio de conservação da integridade pessoal}

Privacidade preservada: ( ) sim ( ) não

Especificar.

Crença religiosa:

O paciente faz parte nas decisões do tratamento: ( ) sim ( ) não

Quais:

Grau de dependência: ( ) total ( ) parcial ( ) independente

Especificar:

O paciente conhece a unidade de centro cirúrgico: ( ) Sim ( ) Não

Observações:.

Sentimentos do paciente: ( ) impressão ( ) expressão

( ) calma ( ) apatia ( ) angústia ( ) medo ( ) negação ( ) agitação

( ) isolamento

Observações:.....

Conhecimento do paciente em relação ao procedimento cirúrgico:

( ) nada ( ) pouco ( ) suficiente ( ) muito

Observações:.

Conhecimento do paciente em relação ao procedimento anestésico:

( ) nada ( ) pouco ( ) suficiente ( ) muito

Observações:.

\subsection{Dados relacionados ao princípio de conservação da integridade social}

Presença da família: ( ) sim ( ) não

Elo de ligação

(parentesco).

A família participa nos cuidados ao paciente no hospital: ( ) sim ( ) não

Observações. 
PICCOLI, Marister; GALVÃO, Cristina Maria. BIOÉTICA - UM ENSAIO SOBRE SUA INSERÇÃO NOS CURSOS DE GRADUAÇÃO EM ENFERMAGEM. Revista Eletrônica de Enfermagem, v. $07, \quad$. $03, \quad$ p. 366 - $372, \quad 2005$. Disponível em http://www.fen.ufg.br/Revista/revista7 3/atualizacao.htm

O que a família sabe em relação ao procedimento cirúrgico que o paciente será submetido: ( ) nada ( ) pouco

( ) suficiente ( ) muito

Observações.......

O que a família sabe em relação ao procedimento anestésico que o paciente será submetido:

( ) nada ( ) pouco ( ) suficiente ( ) muito

Observações.......

Houve mudança na sua vida pessoal devido à necessidade do procedimento cirúrgico:

( ) $\operatorname{sim}$ ( ) Não Quais:.

Houve mudanças na vida profissional devido à necessidade do procedimento cirúrgico:

( ) Sim ( ) Não Quais..

\section{CONSIDERAÇÕES FINAIS}

De acordo com LEONARD (1993), o modelo conceitual de Levine equipara-se a muitos elementos do processo de enfermagem e supõe que enfermeiro e paciente participem juntos do planejamento da assistência de enfermagem. Conforme relata o autor, para Levine na fase de avaliação podemos utilizar dois métodos, a entrevista e a observação, onde a atenção recai sobre a família e outros, caso essas pessoas sejam importantes para o paciente e afirma ainda que Levine, na abordagem integral faz uso dos quatro princípios de conservação como guia na avaliação, onde o enfermeiro preocupa-se com o equilíbrio de energia, com a manutenção da integridade estrutural, pessoal e social e assim coletará dados a respeito desses quatro princípios.

Após esta fase LEONARD (1993) relata que Levine indica uma análise crítica de modo a obter uma visão integral do paciente, indicando dessa forma, os pontos fracos e fortes do paciente de acordo com os quatro princípios de conservação, nesta análise são identificados pontos que necessitam de posterior coleta de dados. A autora acrescenta que para Levine, nesta análise são levados em consideração, normas, conceitos e outras teorias de outras disciplinas.

Ao término do nosso estudo constatamos que o modelo conceitual de Levine utilizado, possibilitou observar a complexidade que envolve o paciente cirúrgico, e na seqüência o processo de assistência da enfermagem perioperatória, os princípios são claros, mas pensamos que eles não devem ser entendidos separadamente, pois algumas necessidades afetadas podem ser encontradas e abordadas em mais de um princípio de conservação.

Salientamos ainda, que o modelo conceitual de Levine considera a família como fator insubstituível na recuperação do paciente, garantindo assim, uma troca mútua, o paciente estará satisfeito por estar ao lado de pessoas que lhe fazem bem e a família também estará satisfeita em poder participar na recuperação do paciente.

Uma sugestão é que este instrumento possa ser utilizado como um roteiro de visita pré-operatória para o enfermeiro de centro cirúrgico, o qual considera a individualidade de cada um e ao mesmo tempo direciona a identificação das necessidades do paciente que possam interferir durante o procedimento cirúrgico.

A presente investigação nos levou a compreensão da importância de utilizarmos um modelo conceitual para fundamentar a sistematização da assistência de enfermagem que melhor se adeque à realidade de cada situação, neste caso, a enfermagem perioperatória.

Ressaltamos que o modelo selecionado pode levar a viabilidade da sistematização e não a uma sobrecarga de serviço, mas sim como forma de organização, sendo que a prioridade é o paciente, assim, pensamos ser este instrumento também um facilitador de informações, reforçando a cientificidade na prática da assistência de enfermagem perioperatória e a responsabilidade do enfermeiro, levando a autonomia da profissão.

Consideramos que este estudo atendeu os seus objetivos, no que diz respeito à elaboração de um roteiro de visita pré-operatória que serviu como guia de coleta de dados através da visita pré-operatória de enfermagem tendo em vista as necessidades do transoperatório, demonstrou que o modelo conceitual de Levine pode ser aplicado à prática profissional.

Entendemos que os quatro princípios de conservação de Levine abrangem o paciente como um todo, e principalmente não só a ele, mas aqueles que são importantes para ele, e auxiliarão na recuperação do que seja a totalidade para cada paciente.

Com este trabalho esperamos destacar a importância da visita pré-operatória de enfermagem pelo enfermeiro de centro cirúrgico, entretanto faz-se necessário que este profissional incorpore em seu diaa-dia a utilização de um instrumento de coleta de dados que possibilite a identificação dos diagnósticos de enfermagem que possam fornecer a base para o planejamento, só assim poderemos promover melhora da prática profissional e consequentemente, o paciente terá assistência integral e individualizada, e principalmente humanizada.

A partir da sistematização da assistência de enfermagem perioperatória, o enfermeiro pode implementar intervenções fundamentadas em princípios científicos, e ao nosso ver, a participação do paciente e sua família deve ocorrer do começo ao fim do processo terapêutico. 
PICCOLI, Marister; GALVÃO, Cristina Maria. BIOÉTICA - UM ENSAIO SOBRE SUA INSERÇÃO NOS CURSOS DE GRADUAÇÃO EM ENFERMAGEM. Revista Eletrônica de Enfermagem, v. 07, $n . \quad 03, \quad$ p. 366 - 372, $2005 . \quad$ Disponível em http://www.fen.ufg.br/Revista/revista7 3/atualizacao.htm

\section{REFERÊNCIAS BIBLIOGRÁFICAS}

CASTELLANOS, B.E.P. Aplicação do processo de enfermagem na unidade de centro cirúrgico. Rev. Esc. Enfermagem USP, São Paulo, v.12, n.3, p.170-86, 1978.

CASTELLANOS, B.E.P.; MANDELBAUM, M.H.S. Uma proposta para discussão: o papel do enfermeiro na unidade de centro cirúrgico. Rev. Paul. Enfermagem, São Paulo, v.5, n.1, p.15-20, jan./mar. 1985.

LEONARD, M.K. Myra Strin Levine. In: GEORGE, J.B. et al. Teorias de enfermagem: os fundamentos para a prática profissional. Porto Alegre, Artes Médicas, 1993. cap.12, p.164-173.

LEVINE, M.E. Introduction to clinical nursing. 2 ed. Philadelphya: F.A. Davis Co, 1973.

Texto original recebido em 30/08/2005

Publicação aprovada em 30/12/2005 